\title{
AICT-AsiaPCR: continuing the education mission during the pandemic
}

\author{
$\left(0 \frac{2}{0}\right)$ \\ Huay Cheem Tan*, MD, PhD; Course Director AICT-AsiaPCR \\ National University Heart Centre, Singapore, Singapore
}

We are living in extraordinary times. The global COVID-19 pandemic has drastically changed the way we live, work, learn and interact. Like all other global meetings, AICT-AsiaPCR, the official scientific meeting of the Asian Pacific Society of Cardiology (APSIC), has pivoted to a virtual platform, a far cry from the inaugural meeting which opened with a big bang in Singapore in 2019.

You may ask why we still need AICT-AsiaPCR, yet another interventional cardiology meeting, of which there are already so many, and when "webinar fatigue" is a buzzword these days. The reason is simple: this meeting is uniquely Asian. It is a meeting created not by one centre, one local society or one country, but by the entire Asia-Pacific region, where representatives from different countries work on the content of the programmes, develop ideas in consultation with one another, and think of how to deliver lessons in the most practical way so that attendees may apply what they have learnt in their practice almost immediately afterwards. In working with the larger PCR family, the AICT-AsiaPCR taps into global expertise to create new knowledge and showcase new skills to all. The three main missions of the AICT-AsiaPCR are (1) to be the platform that represents the entire Asia-Pacific field of endovascular therapy to the rest of the world; (2) to create unique value by showcasing its expertise and knowledge; and (3) to partner with global communities in the field of endovascular therapy based on the complementarity of cultures and respect. AICT-AsiaPCR continues to exert relevance even at the height of the COVID-19 pandemic, as it strives to find new and creative ways to continue the mission of the Society in "Transforming lives through advancing innovation and global partnerships".

With the experience of organising the meeting virtually last year and after participating in EuroPCR 2021, AICT-AsiaPCR 2021 was organised in a new hybrid format. By staying true to its objective of being practical and by encompassing the best of in-person and virtual meetings, it allowed for knowledge and techniques learnt to be immediately transferrable to daily practice for the attendees. In this year's edition, a breakthrough TV studio format with live audience participation alongside virtual global transmission was conceived. The rich and informative content was delivered in a captivating manner to catch and engage audience attention. Due to the increased demand for script writing, preparatory meetings, faculty training, rehearsals and technical coordination, the Course 
Directors spent more time and effort developing the programme compared to past meetings. The end result was content delivered in an interview format with active discussion conducted between the studio and remote participants, as well as live and virtual audience interaction with the faculties. We believe that this format will likely shape the future of all interventional cardiology meetings.

The meeting was conducted over two days with 15 hours of continuous transmission. Three studios based in Singapore, New Delhi, India, and Kuala Lumpur, Malaysia transmitted the live programme. The studios were supported by eight simultaneously connected pods/centres which televised the live programme to a local, larger audience. There were two channels in this meeting. Channel One was the Live Arena Channel where the live broadcast was transmitted, uninterrupted, from the studios and consisted of both real-time and pre-recorded content. Channel Two was the Virtual Essentials Channel, carrying pre-recorded sessions with a live chat interaction feature. Furthermore, all the content was recorded and is available to be played on-demand should the audience wish to watch it again. As physical, hands-on simulation training was limited, it was substituted with "How To" sessions in which experts demonstrated "step-by-step" approaches and performed useful, albeit somewhat uncommon, interventional techniques.

The content for this year's meeting was especially rich, spanning state-of-the-art coronary and structural interventions. These included important contemporary coronary topics which focussed on left main, bifurcation, chronic total occlusion, mechanical circulatory support, physiology and imaging, the knowledge of which differentiates the modern-day interventionist from older generations who grew up with only balloons and stents. The "Innovation Sessions" were particularly fascinating as they provided a peek into the crystal ball of the future of endovascular therapy. The structural intervention programme was developed in tandem with its explosive growth and development in the Asia Pacific region in the last decade. There was sharing of complex transcatheter aortic valve implantation (TAVI) procedural techniques and complications as well as the introduction of the new TricValve to the region, which offers a potential solution for the treatment of patients with severe tricuspid regurgitation. The highly popular session, "Best Cases and Best Abstracts" received an overwhelming number of 104 interesting cases and 33 abstract submissions. Many of these presentations have been invited for publication in AsiaIntervention, the official scientific journal of APSIC, which is poised to be listed in PubMed.

It has been said that when climbing a mountain, one has to look back to realise how high you have climbed. AICT started in 2005 as a small meeting but has evolved over the years to become the current AICT-AsiaPCR, a meeting which has earned international recognition and importance. There is no question that we will strive to remain nimble and resilient as we adapt to a fast changing world with continuous curveballs being hurled at us.

\section{Conflict of interest statement}

The author has no conflicts of interest to declare. 